\title{
A large single-institution retrospective analysis of aggressive B-cell lymphomas according to the 2016/2017 WHO classification
}

\author{
${ }^{*}$ Dorota Jesionek-Kupnicka ${ }^{1, A-D, F}$, ${ }^{*}$ Marcin Braunn ${ }^{1,2, A-D, F}$, Tadeusz Robak ${ }^{3, E, F}$, Wojciech Kuncmann ${ }^{1, E, F}$, Radzislaw Kordek ${ }^{1, E, F}$ \\ ${ }^{1}$ Department of Pathology, Chair of Oncology, Medical University of Lodz, Poland \\ 2 Postgraduate School of Molecular Medicine, Medical University of Warsaw, Poland \\ ${ }^{3}$ Department of Hematology, Medical University of Lodz, Poland \\ A - research concept and design; B - collection and/or assembly of data; $\mathrm{C}$ - data analysis and interpretation; \\ $\mathrm{D}$ - writing the article; $\mathrm{E}$ - critical revision of the article; $\mathrm{F}$ - final approval of the article
}

\section{Address for correspondence}

Marcin Braun

E-mail: braunmarcin@gmail.com

Funding sources

None declared

Conflict of interest

None declared

* These authors contributed equally to this work.

Received on August 27, 2018

Reviewed on March 10, 2019

Accepted on May 7, 2019

Published online on September 12, 2019

Cite as

Jesionek-Kupnicka D, Braun M, Robak T, Kuncman W, Kordek R. A large single-institution retrospective analysis of aggressive B-cell lymphomas according to the 2016/2017 WHO classification. Adv Clin Exp Med. 2019;28(10):1359-1365. doi:10.17219/acem/109200

DOI

10.17219/acem/109200

Copyright

Copyright by Author(s)

This is an article distributed under the terms of the

Creative Commons Attribution Non-Commercial License

(http://creativecommons.org/licenses/by-nc-nd/4.0/)

\begin{abstract}
Background. High-grade B-cell lymphomas (HGBLs) comprise a new entity in the revised 2016/2017 World Health Organization Classification of Tumours of Haematopoietic and Lymphoid Tissues. The diagnosis of HGBL encompasses histopathology and immunohistochemistry, with additional molecular examination of the $B C L 2 / M Y C$ or $B C L 6 / M Y C$ rearrangement status.
\end{abstract}

Objectives. The aim of the study was to summarize our experience in the histopathological and immunohistochemical diagnosis of patients with aggressive B-cell lymphomas according to the revised 2016/2017 WHO classifications.

Material and methods. We reviewed our single-institution experience with accurate diagnoses of HGBL and diffuse large B-cell lymphoma (DLBCL) using the available histopathological and immunohistochemical tools. The timeframe was from January 1, 2017 to April 18, 2018.

Results. Out of 265 patients, 217 (81.9\%) were diagnosed with DLBCL, 43 (16.2\%) with HGBL/DLBCL and 5 (1.9\%) with not otherwise specified HGBL (HGBL-NOS). Regarding concurrent expression of MYC and $B C L 2$ and/or BCL6 (double expressors (DE) and triple expressors (TE)), more DE and TE cases were found in the HGBL/DLBCL group than in the DLBCL group (25.53\% vs $8.47 \%, p<0.001$, for DE cases and $55.32 \%$ vs $6.21 \%, p<0.001$, for TE cases). All 48 (100.00\%) of the HGBL-NOS and HGBL/DLBCL patients, and 26 $(11.98 \%)$ of the DLBCL-DE/TE cases were recommended for molecular analysis.

Conclusions. Our findings show that a comprehensive histopathological and immunohistochemical examination may identify potential HGBL cases. This study emphasizes the need to introduce a suitable molecular examination for patients with HGBL morphology and/or double/triple expression of BCL2/BCL6/MYC proteins.

Key words: DLBCL, World Health Organization (WHO) 2016/2017 Classification Of Tumours Of Haematopoietic and Lymphoid Tissues, DLBCL/BL, HGBL, high grade B-cell lymphoma/diffuse large B-cell lymphoma 
The revised 2016/2017 World Health Organization (WHO) Classification of Tumours of Haematopoietic and Lymphoid Tissues included new entities among aggressive B-cell lymphomas. ${ }^{1,2}$ The most significant changes were introduced by distinguishing a new category of high grade B-cell lymphomas (HGBLs), which were mainly derived from previous provisional categories, including unclassifiable B-cell lymphomas with features intermediate between diffuse large B-cell lymphoma (DLBCL) and Burkitt's lymphoma (BL) (BCLU), as well as from DLBCLs of classic morphology. ${ }^{1,2}$

The HGBL category was created based on the differences between its pathogenesis and clinico-pathological features and those of DLBCL or BL. ${ }^{2-4}$ The poorer prognosis among HGBL patients in comparison to those with DLBCL or BL established a strong need to develop novel, more intensive therapies, as the standard regimen of rituximab, cyclophosphamide, vincristine, and doxorubicin (R-CHOP) is ineffective in HGBL patients. ${ }^{3,5-11}$ Although this new category is still highly heterogeneous, it creates an opportunity for a more accurate diagnosis and may ultimately lead to better treatment allocation or facilitate the search for new treatment strategies in randomized clinical trials.

An accurate diagnosis of HGBL encompasses standard histopathological and immunohistochemical analyses combined with molecular examination. ${ }^{2,4}$ Recently, Szumera-Ciećkiewicz et al. comprehensively summarized the current practical guidelines on differential diagnosis of aggressive B-cell lymphomas, including the proper differentiation of HGBL. ${ }^{4}$ In brief, the current protocols for DLBCL/HGBL/BL diagnosis require the following to be determined: 1) the morphology of the lymphoma cells; 2) the cell of origin (COO), using the immunohistochemistry-based Hans protocol; 3) an immunohistochemical analysis of BCL2, BCL6 and MYC expression, as well as Tdt and cyclin D1/SOX11 in cases with blastoid morphology; and 4) a fluorescent in situ examination of BCL2/MYC or BCL6/MYC rearrangements (Table 1). ${ }^{1,2,4}$ While the first
3 requirements are widely available in Polish histopathology laboratories, there is a deficiency in access to proper molecular examinations.

In this study we aimed to conduct a large retrospective single-institutional report on the morphological and immunohistochemical diagnosis of aggressive B-cell lymphomas in light of the revised 2016/2017 World Health Organization (WHO) Classification of Tumours of Haematopoietic and Lymphoid Tissues.

The work described in this article was carried out in accordance with the Code of Ethics of the World Medical Association (Declaration of Helsinki) for experiments involving humans; EU Directive 2010/63/EU for animal experiments; and uniform requirements for manuscripts submitted to biomedical journals.

\section{Patients and methods}

\section{Study cohort}

All aggressive B-cell lymphoma cases diagnosed at the Department of Pathology, Chair of Oncology, Medical University of Lodz, Poland, between January 1, 2017 and April 18, 2018 were included in the analysis. The review was restricted to cases classified as HGBL not otherwise specified (HGBL NOS - cases with clear blastoid morphology or not meeting the criteria for standard DLBCL or BL - Table 1), DLBCL and HGBL/DLBCL (a category for cases which in histopathological and immunohistochemical analysis appear to be HGBL, but no molecular confirmation was accessible). Formalin-fixed paraffin-embedded (FFPE) samples were collected, along with hematoxylin and eosin (H\&E) and immunohistochemical slides. Finally, pathological data obtained from the patients was extracted into a pre-prepared Excel (v. 1907, Office 365; Microsoft Corp., Redmond, USA) spreadsheet. The required data encompassed the patient's age at diagnosis, sex, diagnosis,

Table 1. High grade B-cell lymphoma (HGBL) subtypes and diagnostic criteria according to the 2016/2017 WHO Classification of Tumours of Haematopoietic and Lymphoid Tissues

\begin{tabular}{|c|c|c|c|c|}
\hline Subtype & Morphology features & Immunohistochemistry & $\begin{array}{l}\text { FISH analysis for } B C L 2 / M Y C \\
\text { or } B C L 6 / M Y C \text { rearrangements }\end{array}$ & $\begin{array}{l}\text { Most common diagnosis } \\
\text { according to the previous } \\
2008 \text { WHO classification }\end{array}$ \\
\hline HGBL, NOS & $\begin{array}{l}\text { Mandatory for diagnosis; } \\
\text { blastoid or Burkitt-like } \\
\text { (between BL and DLBCL) }\end{array}$ & $\begin{array}{c}\text { GCB to ABC ratio 2:1; } \\
\text { DE/TE - most cases; } \\
\text { negative for TdT and cyclin D1/SOX11 }\end{array}$ & Not mandatory for diagnosis & $\mathrm{DLBCL} / \mathrm{BL}$ \\
\hline $\begin{array}{l}\mathrm{HGBL}, \\
\mathrm{DH} / \mathrm{TH}\end{array}$ & $\begin{array}{l}\text { Not mandatory for diagnosis; } \\
\text { DLBCL - most cases, blastoid } \\
\text { or Burkitt-like (between BL } \\
\text { and DLBCL) }\end{array}$ & $\begin{array}{c}\text { GCB - most cases, } \\
\text { DE/TE - most cases, } \\
\text { negative for TdT and cyclin D1/SOX11 }\end{array}$ & $\begin{array}{l}\text { Mandatory for diagnosis; } \\
\text { positive for } B C L 2 / M Y C \text { or } B C L 6 / \\
\text { MYC rearrangement present }\end{array}$ & $\mathrm{DLBCL}$ \\
\hline $\begin{array}{l}\text { HGBL, DH/TH, } \\
\text { transformed } \\
\text { from FL }\end{array}$ & $\begin{array}{l}\text { Mandatory for diagnosis; } \\
\text { history of follicular } \\
\text { lymphoma, } \\
\text { DLBCL - most cases }\end{array}$ & $\begin{array}{c}\text { GCB - most cases, } \\
\text { DE/TE - most cases, } \\
\text { negative for TdT and cyclin D1/SOX11 }\end{array}$ & $\begin{array}{l}\text { Mandatory for diagnosis; } \\
\text { positive for } B C L 2 / M Y C \text { or } B C L 6 / \\
\text { MYC rearrangement present }\end{array}$ & DLBCL \\
\hline
\end{tabular}

NOS - not otherwise specified; DH - double-hit; TH - triple hit; GCB - germinal center B-cell phenotype; ABC - activated B-cell phenotype; DE - double expressor; TE - triple expressor; FISH - fluorescent in-situ hybridization; DLBCL/BL - unclassifiable B-cell lymphomas with features intermediate between diffuse large B-cell lymphoma (DLBCL) and Burkitt's lymphoma (BL). 
morphology, the results of immunohistochemical analysis including $\mathrm{COO}$ classification, Ki67 proliferation index, as well as BCL2, BCL6 and MYC expression.

\section{Pathological diagnostic approach}

Since January 1, 2017, our institution has followed a new diagnostic protocol for all aggressive B-cell lymphomas. The protocol is in accordance with the 2016/2017 WHO Classification of Tumours of Haematopoietic and Lymphoid Tissues. In this protocol a diagnostic report of a lymphoma encompasses: 1) morphological texture (e.g., "high grade B-cell lymphoma with blastoid morphology”); 2) immunophenotype along with $\mathrm{Ki} 67$ proliferation index; 3) COO: germinal center B-cell phenotype (GCB) or activated $\mathrm{B}$-cell phenotype (ABC), according to the Hans protocol $^{12}$; 4) the status of immunoexpression of BCL2, BCL6 and/or MYC proteins (double expressor, triple expressor, non-double expressor); 5) a conclusion, including an indication whether the patient needs an additional molecular examination (e.g., "According to the 2016/2017 WHO
Classification of Tumours of Haematopoietic and Lymphoid Tissues, an additional fluorescence in situ hybridization (FISH) analysis should be performed on the sample for differential diagnosis between DLBCL and HGBL, $\mathrm{DH} / \mathrm{TH}$ "). Examples of HGBL/DLBCL cases are shown in Fig. 1,2.

\section{Immunohistochemistry}

The standard panel of antibodies examined in patients with DLBCL, BL and HGBL covered CD20, CD3, BCL2, BCL6, MYC, CD10, MUM1, Ki67, cyclin D1, SOX11, TdT, CD5, CD38, and PAX5 (BSAP). Clones of the antibodies along with the manufacturers are listed in Table 2. Immunohistochemical analysis used monoclonal antibodies (FLEX Monoclonal Mouse Anti-Human, Dako A/S, Glostrup, Denmark) and EnVisionTMFLEX+ (Dako A/S) for the visualization. The tests were carried out using Autostainer Link 48 (Dako A/S).

The expression for all markers was reported as positive or negative, but for BCL2, BCL6, MYC, and Ki67 detailed

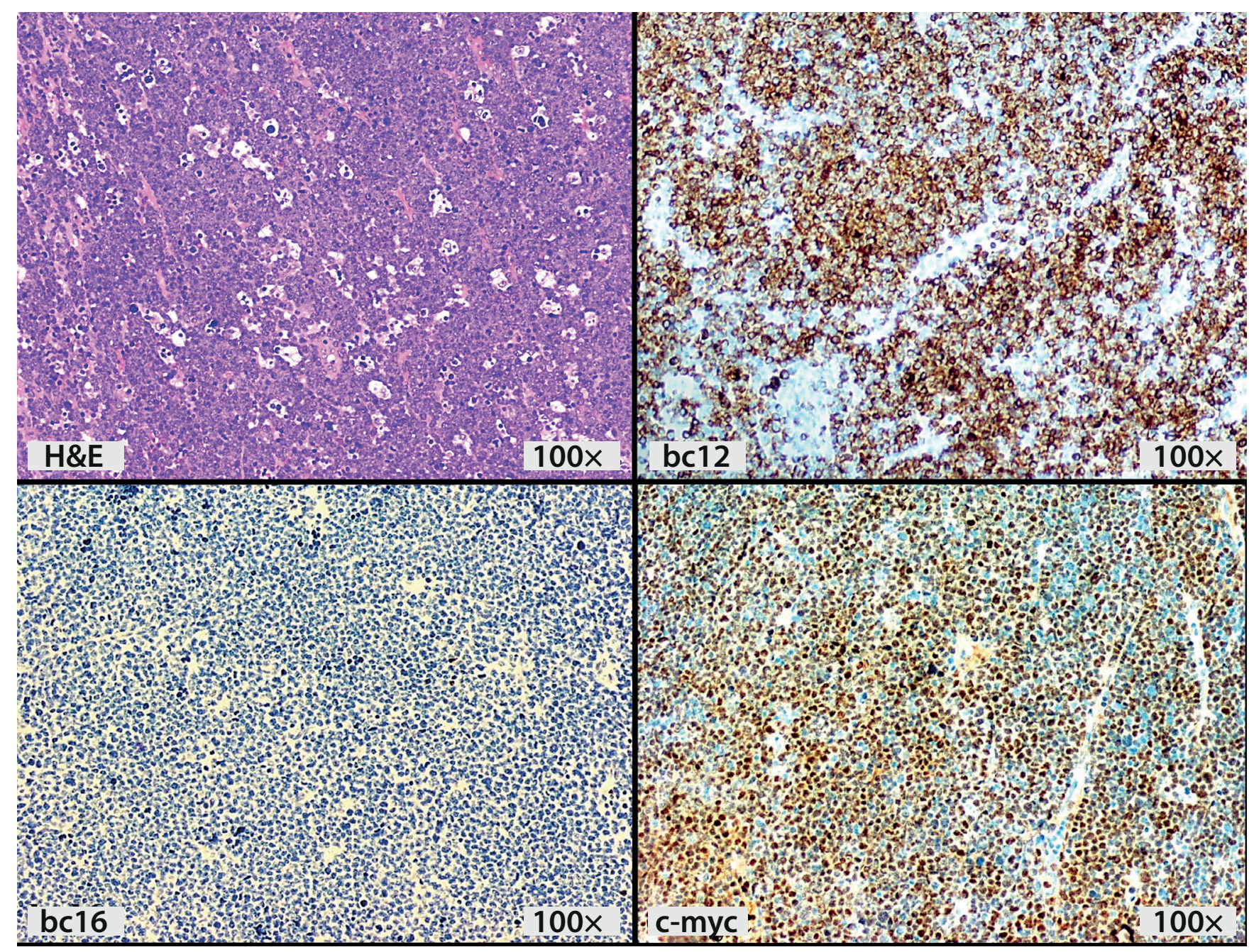

Fig. 1. Histopathological example of a high grade B-cell lymphoma not otherwise specified (HGBL NOS) with the starry-sky pattern. The case was of germinal center B-cell phenotype origin and revealed BCL2 and MYC expression without expression of BCL6 


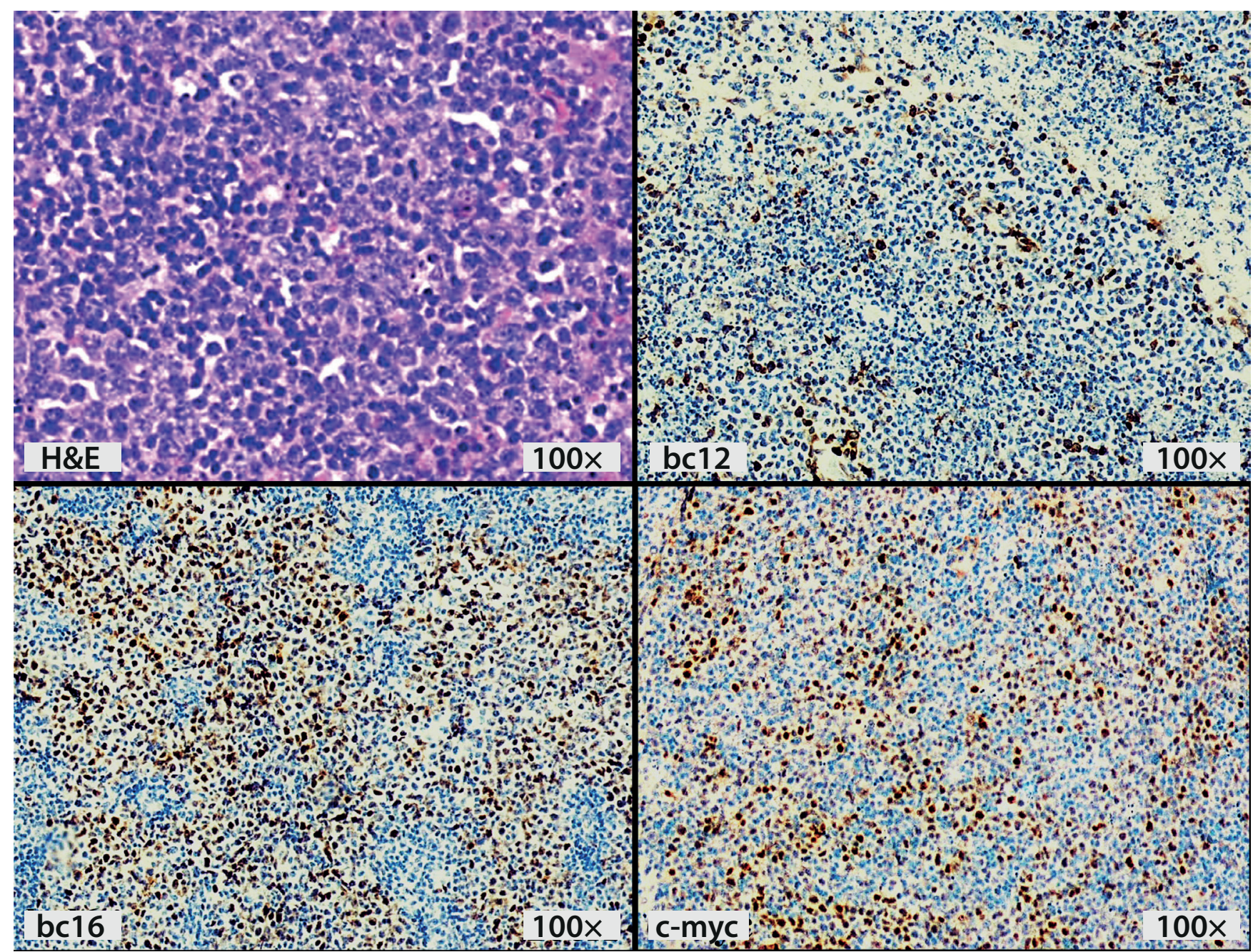

Fig. 2. Histopathological example of a high grade B-cell lymphoma not otherwise specified (HGBL NOS) with blastoid morphology. The case was of germinal center B-cell phenotype, revealed BCL6 and MYC expression without expression of BCL2 and was negative for TdT

DE - double expressor

Table 2. List of antibodies used in the immunohistochemical analysis

\begin{tabular}{|l|c|c|}
\hline \multicolumn{1}{|c|}{ Antibody } & Clone & Company \\
\hline BCL2 & 124 & Dako \\
\hline BCL6 & PG-B6p & Dako \\
\hline BSAP (PAX-5) & DAK-Pax5 & Dako \\
\hline CD3 & Polyclonal rabbit & Dako \\
\hline CD5 & $4 C 7$ & Dako \\
\hline CD10 & 56C6 & Dako \\
\hline CD20 & L26 & Dako \\
\hline CD38 & SP149 & Cell Marque \\
\hline Cyclin D1 & EP12 & Dako \\
\hline MYC & Y69 & VENTANA \\
\hline Ki-67 & MIB-1 & Dako \\
\hline MUM-1 & MUM1p & Dako \\
\hline SOX-11 & MRQ-58 & Cell Marque \\
\hline TdT & EP266 & Dako \\
\hline
\end{tabular}

percentages of positive cells were reported in brackets. Cases were marked as positive under the following conditions: when more than $50 \%$ of the lymphoma cells were stained with anti-BCL2 antibody (cytoplasmic and nuclear staining), when more than $30 \%$ of the lymphoma cells were stained with BCL6 (nuclear staining) or when more than $40 \%$ of the lymphoma cells were stained with MYC (nuclear staining). The immunohistochemistry results were validated using positive and negative tissue controls in the whole series of immunostained slides.

\section{Statistical analysis}

Continuous variables were presented as medians followed by interquartile range (IQR), while nominal variables were presented as numbers followed by percentages in brackets. The Shapiro-Wilk test was used to assess the normality of distribution. Continuous variables were compared using the Mann-Whitney U test in case of nonnormal distribution. Differences between categorical 
variables were evaluated using the $X^{2}$ or two-tailed Fisher's exact tests. The STATISTICA v. 12.5 PL software package (Statsoft Inc., Tulsa, USA) was used for the analysis. P-values $<0.05$ were considered statistically significant.

\section{Results}

\section{Patients characteristics}

Between January 1, 2017 and April 18, 2018, 265 patients were diagnosed with either DLBCL $(\mathrm{n}=217,81.88 \%)$, HGBL, NOS ( $\mathrm{n}=5,1.89 \%$ ), or HGBL/DLBCL ( $\mathrm{n}=43$, $16.23 \%$ ). The median age of the whole group was 69 years $(\mathrm{IQR}=61.12-77.77)$. The HGBL/DLBCL patients were older than DLBCL patients; however, this result was not statistically significant. Almost $1 / 2$ of the patients in each group were males.

All HGBL NOS cases presented a blastoid-pattern morphology and were either double or triple expressors. Due to the small number of cases, all 5 HGBL NOS cases were included with the HGBL/DLBCL patients. The details of the study group, along with comparisons between the DLBCL and HGBL/DLBCL patients, are presented in Table 3.

\section{Lymphoma morphology}

Within the HGBL/DLBCL group, 6 patients (12.50\%) presented with partially blastoid morphological features, and 14 patients $(29.17 \%)$ presented with BL morphological features, while more than $1 / 2$ presented with standard DLBCL morphology (mainly centroblastic or immunoblastic).

\section{Comparison of cell of origin of lymphomas and Ki67 index}

The majority of the lymphomas displayed an immunophenotype characteristic of ABCs. This tendency was stable regardless of the study group. There were $33 \mathrm{ABCs}(70.21 \%)$ in the HGBL/DLBCL group and $122 \mathrm{ABCs}$ (59.80\%) in the DLBCL group ( $\mathrm{p}=0.186)$.

The Ki67 proliferation index in the whole study group was high: $90 \%$ (IQR = 80-95\%). It differed significantly between HGBL/DLBCL and DLBCL patients: 95\% (90-100\%) vs $90.00 \%$ (75-90\%), respectively ( $\mathrm{p}<0.001)$.

\section{Comparison of $\mathrm{BCL} 2, \mathrm{BCL} 6$ and MYC expression}

The majority of the lymphomas studied displayed positivity for BCL2 and BCL6 expression: $\mathrm{n}=156$ (65.27\%) and $\mathrm{n}=171$ (67.86\%), respectively; and 67 patients (30.04\%) were positive for MYC expression. The percentage of BCL6-positive lymphomas did not differ significantly between the study groups $(\mathrm{p}=0.466)$. However, HGBL/DLBCL cases were more frequently BCL2-positive and MYC-positive than DLBCL cases: 41 (87.23\%) vs 115 (59.90\%) BCL2-positive cases $(\mathrm{p}<0.001)$ and 38 (80.85\%) vs 29 (16.48\%) MYCpositive cases $(\mathrm{p}<0.001)$.

Regarding concurrent expression of MYC and BCL2 and/or BCL6, more DE and TE cases were found in the HGBL/DLBCL group than in the DLBCL group: 12 (25.53\%) vs $15(8.47 \%)$ DE cases $(\mathrm{p}<0.001)$ and 26 (55.32\%) vs 11 (6.21\%) TE cases ( $\mathrm{p}<0.001)$; DE and TE cases comprised $54(20.40 \%)$ of the patients in the study.

\section{Patients recommended for molecular analysis}

We recommended fluorescence in situ hybridization (FISH) analysis for identifying $B C L 2 / M Y C$ and
HGBL - high grade B-cell lymphoma; NOS - not otherwise specified; BL - Burkitt's lymphoma; GCB - germina applicable; KMH - Kopernik Memorial Hospital; EC - external oncological centers; DE - double expressor; TE - triple expressor 
BCL6/MYC rearrangements in all 48 (100\%) of the HGBL/ DLBCL cases and in 26 (11.98\%) of the DLBCL cases, i.e., 74 (27.92\%) of all the patients included in the study. As of the end of this study, none of the patients had been sent for molecular analysis.

\section{Discussion}

During the 18 months following the introductions of the revised 2016/2017 WHO Classification of Tumors of Haematopoietic and Lymphoid Tissues, our clinic recommended almost $20 \%$ of HGBL patients and $30 \%$ of HGBL/DLBCL-DE patients for FISH analysis for proper discrimination between HGBL, DH/TH and DLBCL. Considering the important clinical and biological differences between these diagnoses, we demonstrated a pressing need to apply the differential diagnosis of DLBCL and HGBL in the routine diagnosis of lymphomas in Poland.

The HGBL category was formally introduced in 2017 with the publication of the new WHO classifications; however, this distinct subgroup of lymphomas had been proposed a few years earlier. ${ }^{2,4,11}$ The most important morphological criterion (the blastoid pattern) and the additional immunohistochemical criteria (double- or triple- expression of MYC, BCL2 and/or BCL6) are most often published in guidelines and reviews. ${ }^{3,4}$ Therefore, the present study separated all patients who were positive for the first or both criteria and labelled them as HGBL/DLBCL patients. No stringent criteria exist that can help to determine the additional molecular testing needed to separate HGBL DH/TH cases. ${ }^{1,3,4,13,14}$

HGBL/DLBCL patients constituted almost $20 \%$ of our study group. This percentage is a little lower than literature values (in the largest cohorts, $30 \%$ of DLBCL cases were classified as HGBL/DLBCL; 6\% were confirmed as HGBL-DH after molecular testing). ${ }^{2-4}$ In our study, the discrepancy was eliminated when DLBCL DE/TE cases were included in the group recommended for additional testing. ${ }^{2-4}$ The inclusion of DLBCL DE/TE for additional molecular assessment is clearly substantiated by recent reports on DLBCL DH cases within the DLBCL DE/TE group. $3,4,15,16$

Most of the morphological and immunohistochemical characteristics identified in the group were consistent with those presented in previous reports; however, 2 were found to be intriguingly different. Firstly, almost $70 \%$ of the HGBL/DLBCL group displayed an ABC subtype phenotype when we assessed the COO using the Hans algorithm. The opposite GCB-to-ABC ratio is typically presented in the literature, with the value reaching as high as $100 \%$ GCB in true HGBL DH (BCL2/MYC rearranged) cases. ${ }^{4}$ The older age of the HGBL/DLBCL patients in our study (median age: 72 years) may explain this discrepancy, as $\mathrm{ABC}$ cases are more common among older patients. ${ }^{4}$ Another possible explanation is that our sample included a lower percentage of DE/TE cases among the ABC DLBCLs than reported previously. ${ }^{3,5,7}$ Secondly, while a significantly higher Ki67 proliferation index was found in the HGBL/ DLBCL group than in the DLBCL group in the present study, previous reports indicate no significant differences in this parameter, and advise against using it as a differential criterion for HGBL and DLBCL cases. ${ }^{1,2,4,5}$ We agree with these observations, because despite the statistical significance, the absolute differences in $\mathrm{Ki} 67$ proliferation index in our study were around 5\%.

The need for detection of HGBL is reinforced not only by the different pathogenesis of HGBL and DLBCL, but also by the important clinical differences between HGBL and DLBCL mentioned previously. ${ }^{1,3}$ In comparison with DLBCL patients, HGBL (DH/TH or NOS) patients are characterized by shorter overall and event/progression-free survival, and are more frequently associated with poor prognostic factors, such as age at diagnosis, high IPI scores and advanced disease. ${ }^{3,4}$ Moreover, diagnoses of HGBL (DH/ TH or NOS) may soon become predictive for treatment allocation, especially among younger patients; most studies indicate that this group should be treated more intensively than with a standard $\mathrm{R}-\mathrm{CHOP}$ regimen. ${ }^{3,5,17,18}$ However, it must be emphasized that trials of more intensive or novel regimens in HGBL have reported inconsistent results, and the evidence is still not strong enough to prepare distinct treatment guidelines for HGBL patients. ${ }^{2,3,15,19-23}$ This further emphasizes the need for better distinction of HGBL as a highly heterogeneous, provisional category of lymphomas, which should be investigated further.

The major limitation of our study is its lack of FISH analysis of HGBL/DLBCL and DLBCL DE/TE cases, which might be valuable for the final diagnosis. We plan to conduct this type of examination whenever possible in the future. A second limitation concerns the limited amount of clinical data and follow-up. We did not present these data in this report because the scope of the study was restricted to the diagnostic aspect of HGBL/DLBCL. In addition, our pathology department treats patients from several regional oncology centers and gathering such a large volume of data would be excessively time-consuming. Finally, the patients in our study were diagnosed following January 1, 2017 and no accurate conclusions could be drawn in such a short time since the diagnosis. We plan to update the report with clinical and molecular details in the near future.

\section{Conclusions}

Our findings show that comprehensive histopathological and immunohistochemical examinations can identify potential HGBL cases. As many as $20 \%$ of our HGBL/ DLBCL patients would need FISH examination for BCL2/ $M Y C$ or $B C L 6 / M Y C$ rearrangements. This is the strongest justification for the need to introduce appropriate examinations among patients with high grade B-cell lymphomas. 


\section{ORCID iDs}

Dorota Jesionek-Kupnicka (D) https://orcid.org/0000-0001-9319-9570 Marcin Braun (D) https://orcid.org/0000-0003-3804-7042 Tadeusz Robak (D) https://orcid.org/0000-0002-3411-6357 Wojciech Kuncman (D) https://orcid.org/0000-0003-1117-4463 Radzislaw Kordek (D) https://orcid.org/0000-0003-4724-3627

\section{References}

1. Swerdlow S, Campo E, Harris N, et al. WHO Classification of Tumours of Haematopoietic and Lymphoid Tissues. Revised $4^{\text {th }}$ ed. Lyon, France: IARC Press; 2017.

2. Swerdlow SH, Campo E, Pileri SA, et al. The 2016 revision of the World Health Organization classification of lymphoid neoplasms. Blood. 2016;127(20):2375-2390. doi:10.1182/blood-2016-01-643569

3. Sesques $P$, Johnson NA. Approach to the diagnosis and treatment of high-grade B-cell lymphomas with MYC and BCL2 and/or BCL6 rearrangements. Blood. 2017;129(3):280-288. doi:10.1182/blood2016-02-636316

4. Szumera-Ciećkiewicz A, Rymkiewicz G, Grygalewicz B, et al. Comprehensive histopathological diagnostics of aggressive B-cell lymphomas based on the updated criteria of the World Health Organization's 2017 classification. Polish J Pathol. 2017;69(1):1-19. doi:10.5114/ PJP.2018.75332

5. Johnson NA, Slack GW, Savage KJ, et al. Concurrent expression of MYC and $B C L 2$ in diffuse large B-cell lymphoma treated with rituximab plus cyclophosphamide, doxorubicin, vincristine, and prednisone. J Clin Oncol. 2012;30(28):3452-3459. doi:10.1200/JCO.2011.41.0985

6. Green TM, Young KH, Visco C, et al. Immunohistochemical double-hit score is a strong predictor of outcome in patients with diffuse large B-cell lymphoma treated with rituximab plus cyclophosphamide, doxorubicin, vincristine, and prednisone. J Clin Oncol. 2012;30(28): 3460-3467. doi:10.1200/JCO.2011.41.4342

7. Hu S, Xu-Monette ZY, Tzankov A, et al. MYC/BCL2 protein coexpres sion contributes to the inferior survival of activated B-cell subtype of diffuse large B-cell lymphoma and demonstrates high-risk gene expression signatures: A report from the International DLBCL Rituximab-CHOP Consortium Program. Blood. 2013;121(20):4021-4031. doi:10.1182/blood-2012-10-460063

8. Yan L-X, Liu Y-H, Luo D-L, et al. MYC expression in concert with BCL2 and BCL6 expression predicts outcome in Chinese patients with diffuse large B-cell lymphoma, not otherwise specified. PLoS One. 2014; 9(8):e104068. doi:10.1371/journal.pone.0104068

9. Horn H, Ziepert M, Becher C, et al; German High-Grade Non-Hodgkin Lymphoma Study Group. MYC status in concert with BCL2 and BCL6 expression predicts outcome in diffuse large B-cell lymphoma. Blood. 2013;121(12):2253-2263. doi:10.1182/blood-2012-06-435842

10. Perry AM, Alvarado-Bernal Y, Laurini JA, et al. MYC and BCL2 protein expression predicts survival in patients with diffuse large B-cell lymphoma treated with rituximab. Br J Haematol. 2014;165(3):382-391. doi:10.1111/bjh.12763
11. Macpherson N, Lesack D, Klasa R, et al. Small noncleaved, non-Burkitt's (Burkitt-like) lymphoma: Cytogenetics predict outcome and reflect clinical presentation. J Clin Oncol. 1999;17(5):1559-1567. doi:10.1200/ JCO.1999.17.5.1558

12. Hans CP, Weisenburger DD, Greiner TC, et al. Confirmation of the molecular classification of diffuse large B-cell lymphoma by immunohistochemistry using a tissue microarray. Blood. 2004;103(1):275-282. doi:10.1182/blood-2003-05-1545

13. Cheah CY, Oki Y, Westin JR, Turturro F. A clinician's guide to double hit lymphomas. Br J Haematol. 2015;168(6):784-795. doi:10.1111/ bjh.13276

14. Pedersen $M \varnothing$, Gang $A O$, Brown $P$, et al. Real world data on young patients with high-risk diffuse large B-cell lymphoma treated with R-CHOP or R-CHOEP - MYC, BCL2 and BCL6 as prognostic biomarkers. PLoS One. 2017;12(10):e0186983. doi:10.1371/journal.pone.0186983

15. Scott DW, King RL, Staiger AM, et al. High grade B-cell lymphoma with MYC and BCL2 and/or BCL6 rearrangements with diffuse large B-cell lymphoma morphology. Blood. 2018;(131):2060-2064. doi:10.1182/ blood-2017-12-820605

16. Li S, Young KH, Medeiros LJ. Diffuse large B-cell lymphoma. Pathology. 2018;50(1):74-87. doi:10.1016/j.pathol.2017.09.006

17. Howlett C, Snedecor SJ, Landsburg DJ, et al. Front-line, dose-escalated immunochemotherapy is associated with a significant progression-free survival advantage in patients with double-hit lymphomas: A systematic review and meta-analysis. Br J Haematol. 2015;170(4): 504-514. doi:10.1111/bjh.13463

18. Petrich AM, Gandhi M, Jovanovic B, et al. Impact of induction regimen and stem cell transplantation on outcomes in double-hit lymphoma: A multicenter retrospective analysis. Blood. 2014;124(15):2354-2361. doi:10.1182/blood-2014-05-578963

19. Johnson NA, Savage KJ, Ludkovski O, et al. Lymphomas with concurrent BCL2 and MYC translocations: The critical factors associated with survival. Blood. 2009;114(11):2273-2279. doi:10.1182/blood2009-03-212191

20. Puvvada SD, Stiff PJ, Leblanc M, et al. Outcomes of MYC-associated lymphomas after R-CHOP with and without consolidative autologous stem cell transplant: Subset analysis of randomized trial intergroup SWOG S9704. Br J Haematol. 2016;174(5):686-691. doi:10.1111/ bjh.14100

21. Staiger AM, Ziepert M, Horn $\mathrm{H}$, et al; German High-Grade Lymphoma Study Group. Clinical impact of the cell-of-origin classification and the MYC/BCL2 dual expresser status in diffuse large B-cell lymphoma treated within prospective clinical trials of the German High-Grade non-Hodgkin's Lymphoma Study Group. J Clin Oncol. 2017;35(22): 2515-2526. doi:10.1200/JCO.2016.70.3660

22. Rosenthal A, Younes A. High grade B-cell lymphoma with rearrangements of MYC and BCL2 and/or BCL6: Double hit and triple hit lymphomas and double expressing lymphoma. Blood Rev. 2017;31(2):37-42. doi:10.1016/j.blre.2016.09.004 